\title{
ANÁLISE DE PACIENTES DIABÉTICOS ATENDIDOS NO AMBULATÓRIO DE ESPECIALIDADES EM NUTRIÇÃO DA URI- CAMPOS DE FREDERICO
}

\author{
WESTPHALEN- RS
}

\author{
Elis Marina Marchioro ${ }^{1}$ \\ Janice Daiane Brutti2 \\ Rúbia Garcia Deon ${ }^{3}$ \\ Fábia Benetti ${ }^{4}$
}

Recebido em: 14 fev. 2018

Aceito em: 22 nov. 2018

RESUMO: O Diabetes Mellitus (DM) é um grupo heterogêneo de distúrbios metabólicos que tem em comum a hiperglicemia. No Brasil, onde as cidades das regiões Sul e Sudeste são consideradas de maior desenvolvimento econômico, o número de indivíduos que apresentam DM é maior. O objetivo dessa pesquisa foi analisar os pacientes diabéticos atendidos no Ambulatório de Especialidades em Nutrição da URI - Campus de Frederico Westphalen/RS no ano de 2016. Trata-se de um estudo transversal, quantitativo e descritivo. A partir dos prontuários dos pacientes com DM, foram coletados dados pessoais e socioeconômicos, história clínica, hábitos alimentares e medidas antropométricas. Foi identificado que $10 \%$ dos pacientes apresentavam como diagnóstico o DM. Desta amostra, a maioria é do sexo masculino, com média de idade 37,5 $\pm 16,7$ anos. Referente ao Índice de Massa Corporal pode ser observado uma alta frequência de sobrepeso nos pacientes do sexo masculino (55\%), diferentemente do sexo feminino, onde a eutrofia e obesidade I tiveram maior relevância, ambos com 37,5\%. Para fatores de risco, destacou-se uma elevada frequência de pacientes com sobrepeso e obesidade, afetando em torno de $74 \%$ dos pacientes com DM. Já análise do Recordatório Alimentar 24 horas, o consumo de carboidratos apresentou média de $57,6 \pm 10,26 \%$ e proteína $18,68 \pm 9,55 \%$ e são similares os recomendados pela Sociedade Brasileira de Diabetes. Pelo exposto podemos reafirmar a necessidade e importância do acompanhamento nutricional aos pacientes diabéticos, como uma estratégia não farmacológica necessária que beneficiará o paciente no controle da glicemia e no retardo da progressão de comorbidades associadas ao DM.

Palavras-chave: Diabetes Mellitus. Assistência ao Paciente. Avaliação nutricional.

\section{ANALYSIS OF DIABETIC PATIENTS TAKEN IN THE AMBULATORY OF NUTRITION}

\footnotetext{
1 Nutricionista, aluna do Curso de Pós Graduação Nutrição Clínica com Ênfase em Doenças Crônicas e Transtornos Alimentares da Universidade Regional Integrada do Alto Uruguai e das Missões (URI), Departamento de Ciências da Saúde, Setor Nutrição, Frederico Westphalen, Rio Grande do Sul. E-mail: elismarinamarchioro@yahoo.com.br.

${ }^{2}$ Acadêmica do Curso de Nutrição da Universidade Regional Integrada do Alto Uruguai e das Missões-URI Campus de Frederico Westphalen, Departamento de Ciências da Saúde, Setor Nutrição, Frederico Westphalen, Rio Grande do Sul. E-mail: a088878@uri.edu.br.

${ }^{3}$ Nutricionista. Doutora em Gerontologia Biomédica. Docente do Curso de Nutrição da Universidade Regional Integrada do Alto Uruguai e das Missões-URI Campus de Frederico Westphalen, Departamento de Ciências da Saúde, Setor Nutrição, Frederico Westphalen, Rio Grande do Sul. E-mail: rubia@uri.edu.br.

${ }^{4}$ Nutricionista. Mestre em Envelhecimento Humano. Docente do Curso de Nutrição da Universidade Regional Integrada do Alto Uruguai e das Missões-URI Campus de Frederico Westphalen, Departamento de Ciências da Saúde, Setor Nutrição, Frederico Westphalen, Rio Grande do Sul. E-mail: benetti@uri.edu.br.
} 


\section{SPECIALTIES OF URI- CAMPOS DE FREDERICO WESTPHALEN- RS}

ABSTRACT: Diabetes Mellitus (DM) is classified by a heterogeneous group of metabolic disorders that have in common hyperglycemia. More specifically, in Brazil, where the cities of the South and Southeast are considered to be the most economically developed, the number of individuals with DM is higher. In this context, the objective of the present study was to analyze the diabetic patients attended at the Nutrition Specialties Clinic of the URI - Campus of Frederico Westphalen/RS in the year 2016. This is a cross-sectional, quantitative and descriptive study. The study was based on the data collection of patients' records with DM, personal and socioeconomic data were collected, as well as clinical history, eating habits and anthropometric measurements. As a result, it was identified that $10 \%$ of the patients presented DM as their diagnosis. From this sample, the prevalence of patients is male, with a mean age of $37.5 \pm 16.7$ years. Regarding the Body Mass Index, a high frequency of overweight was observed in male patients (55\%), differently from the female sex, where eutrophy and obesity I were more relevant, both with $37.5 \%$. For risk factors, a high frequency of overweight and obese patients was observed, affecting around $74 \%$ of patients with $\mathrm{DM}$, followed by the rate of sedentary and alcoholic patients, in a lower proportion of patients with a diagnosis of Arterial Hypertension (26\%) And smokers with $16 \%$. Already analysis of the Food Recall 24 hours we can verify that the consumption of carbohydrates, with an average of $57.6 \pm 10.26 \%$ and protein $18.68 \pm 9.55 \%$ are similar to those recommended by the Brazilian Society of Diabetes. Therefore, we can reaffirm the need and importance of nutritional monitoring for diabetic patients, as a necessary non-pharmacological strategy that will benefit the patient in glycemic control and delayed progression of comorbidities associated with DM.

Keywords: Diabetes Mellitus. Patient Care. Nutrition assessment.

\section{INTRODUÇÃO}

O Diabetes Mellitus (DM) é caracterizado como uma doença crônica complexa, que necessita cuidados de saúde e que tem como objetivo o controle glicêmico eficaz, a redução de complicações, dos custos financeiros, da morbidade e mortalidade associados à esta patologia (LADE et al., 2016).

É classificado por um grupo heterogêneo de distúrbios metabólicos, que demonstram em comum a hiperglicemia. (TIECHER; NASCIMENTO, 2014). Ele é determinado por concentrações sanguíneas elevadas de glicose que resultam de defeitos na secreção de insulina, ação de insulina, ou ainda em ambos. Quando o DM é diagnosticado, a insulina não é produzida de forma suficiente (MAHAN; ESCOTT-STUMP; RAYMOND, 2012) (TIECHER; NASCIMENTO, 2014).

As complicações do DM podem ser classificadas em agudas ou crônicas. Como complicações agudas existem à hiperglicemia e a hipoglicemia. Já as complicações crônicas podem ser macrovasculares (doença cardíaca coronária, doença vascular periférica e doença cerebrovascular), microvasculares (retinopatia e nefropatia) e neurológicas (neuropatia) (MAHAN; ESCOTT-STUMP, 2013). 
Segundo a Organização Mundial da Saúde (2009) e a Associação Americana de Diabetes (2010), o DM é dividido em quatro classes clínicas: DM tipo I, DM tipo II, outros tipos específicos de DM e DM gestacional. A presença de DM vem crescendo mundialmente, sendo resultante de uma epidemia global. (LADE et al., 2016). Nas Américas, o total de pessoas diagnosticadas com esta patologia foi apurado em 35 milhões para o ano 2000 e projetado para 64 milhões em 2025 (SARTORELLI; FRANCO, 2003).

Há uma predisposição de aumento na frequência em todas as faixas etárias, principalmente nos jovens, onde o impacto negativo sobre a qualidade de vida e a carga da doença aos sistemas de saúde é elevada. Isso ocorre mais especificamente no Brasil, onde as cidades das regiões Sul e Sudeste são consideradas de maior desenvolvimento econômico e o número de indivíduos que apresentam DM é maior (SARTORELLI; FRANCO, 2003).

Conforme a International Diabetes Federation (2013), o Brasil está classificado como o quarto país do mundo em número de indivíduos diabéticos com aproximadamente 12 milhões de doentes.

Nesse contexto, o objetivo do presente estudo foi analisar os pacientes diabéticos atendidos no Ambulatório de Especialidades em Nutrição da URI - Campus de Frederico Westphalen-RS, considerando os fatores de riscos, o Índice de Massa Corporal (IMC) e a análise do Recordatório Alimentar 24 horas destes pacientes.

\section{MATERIAL E MÉTODOS}

Trata-se de um estudo transversal, de cunho quantitativo e de natureza descritiva, desenvolvido como parte do projeto "Acompanhamento Nutricional no Ambulatório de Especialidades em Nutrição da URI - Campus de Frederico Westphalen/RS".

O estudo foi realizado integralmente nas dependências do Ambulatório de Especialidades em Nutrição da URI na cidade de Frederico Westphalen- RS. Os dados foram coletados através da avaliação dos prontuários dos pacientes com DM.

O estudo foi aprovado pelo Comitê de Ética em Pesquisa da Universidade Regional Integrada do Alto Uruguai e das Missões- Campus de Frederico Westphalen, sob o número de registro na Plataforma Brasil CAAE: 53128416.0.0000.5352 e parecer número 1.434.929, (anexo A).

A amostra do estudo foi composta por indivíduos com diagnóstico de DM, Tipo 1 ou 2, através de anamnese alimentar realizada em atendimento nutricional no ambulatório. A seleção dos prontuários ocorreu no período de março de 2016 à fevereiro de 2017. Como critério de exclusão foram considerados os prontuários contendo dados incompletos.

No prontuário dos pacientes diabéticos (anexos B, C e D) atendidos pelo Ambulatório de Especialidades em Nutrição da URI - Campus de Frederico Westphalen/RS, foram coletados dados pessoais do paciente, dados socioeconômicos, 
história clínica, hábitos de vida e hábitos alimentares. Assim como, foram aferidas as medidas antropométricas de peso, estatura, circunferências da cintura, do pescoço e do quadril e também as pregas cutâneas biciptal, triciptal, subescapular e supra ilíaca.

O banco de dados e a análise dos resultados foram feitos no programa Excel 2010. Como forma de análise estatística foi utilizada a estatística descritiva para a apresentação dos dados, que foram expressos em forma de tabela e figura, com percentuais, médias e desvios padrões.

\section{RESULTADOS E DISCUSSÃO}

Foram selecionados 19 (10\%) prontuários, que se enquadraram nos critérios de inclusão, de um total de $182(90 \%)$ pacientes atendidos pelo Ambulatório de Especialidades em Nutrição da URI - Campus de Frederico Westphalen/RS.

Referente ao sexo dos pacientes diabéticos pode ser analisado que 11 indivíduos eram do sexo masculino, sendo estes $58 \%$ dos pesquisados em relação ao sexo feminino que resultou em um número de 8 pacientes, totalizando $42 \%$ da população estudada.

Diferentemente dos resultados encontrados por Lade et al. (2016), onde o maior número dos indivíduos que procuraram o serviço de saúde foram pacientes diabéticos do sexo feminino. Os autores justificam que a participação de mulheres, em maior quantidade que procuram o serviço de nutrição pode ser evidenciada por apresentarem maior tendência ao autocuidado e maior percepção do estado de saúde.

Estes pacientes apresentaram idade entre 9 e 64 anos, tendo como uma média de idade $37 \pm 16,7$ anos. Este resultado pode ser comparado com um estudo realizado por Machado; Campos (2014), onde os autores afirmam que a prevalência a DM na população estudada é de 30 a 69 anos. Idade esta que difere de um estudo realizado por Iser et al (2015), onde o predomínio de DM aumentou com o avanço da idade, sendo que esta se manteve entre de 65 e 74 anos de idade. Outro estudo que reforça que a prevalência de idade acima dos 50 anos é elevada para pacientes diabéticos foi dos autores Cortez et al (2015), que afirmam ainda um crescimento de forma significativa, sendo o DM a doença crônica não transmissível que mais cresce, principalmente nos países em desenvolvimento.

Levando em consideração a patologia apresentada, no caso, o DM, é de extrema importância analisar o Índice de Massa Corporal (IMC) destes pacientes, uma vez que, este fator contribui para o desenvolvimento da doença. Destaca-se o resultado para IMC, sendo: baixo peso $<18,5 \mathrm{~kg} / \mathrm{m}^{2}$, eutrofia $18,5-24,9 \mathrm{~kg} / \mathrm{m}^{2}$, sobrepeso $25-29,9 \mathrm{~kg} / \mathrm{m}^{2}$, obesidade I 30 $34,9 \mathrm{~kg} / \mathrm{m}^{2}$, obesidade II $35-39.9 \mathrm{~kg} / \mathrm{m} 2$, obesidade III $>40 \mathrm{~kg} / \mathrm{m}^{2}$ (Organização Mundial da Saúde, 1998). Este está descrito na Figura 1 por classificação, onde pode ser observada uma alta frequência de sobrepeso nos pacientes do sexo masculino (55\%) assim como a elevada frequência de obesidade III (18\%) e eutrofia (18\%), diferentemente do sexo feminino, onde a eutrofia e obesidade I tiveram maior relevância. 
Figura 1 - Classificação do IMC de pacientes diabéticos

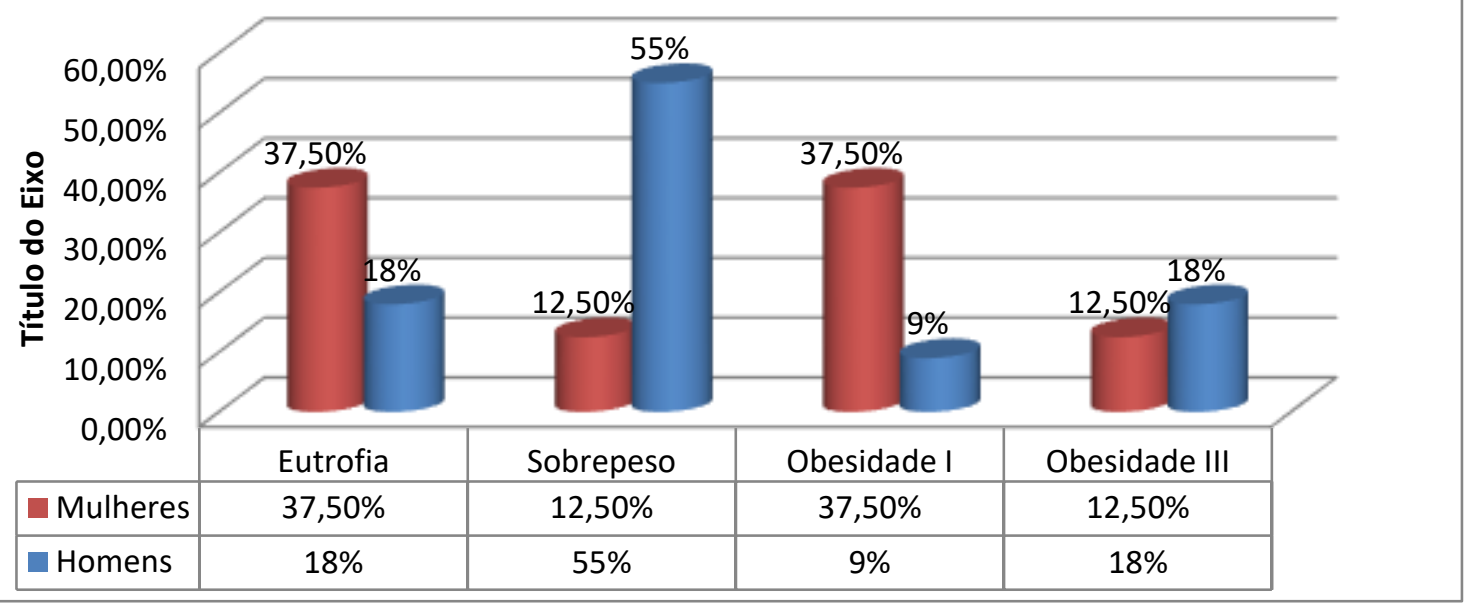

Fonte: MARCHIORO et al, 2017.

Resultado este que corroboram com o de Lade et al (2016), onde o sobrepeso/obesidade apresentado na população estudada demonstrou um alto predomínio representando $72 \%$ nos indivíduos do sexo masculino e $81 \%$ no sexo feminino.

Já no estudo de Flor et al (2015), são demonstradas consistentes associações entre o excesso de peso e a maior prevalência de DM. Essa associação faz-se alarmante em países como o Brasil, onde mais da metade dos indivíduos estão acima do peso ideal.

Existem fatores de risco que aumentam a probabilidade de um indivíduo apresentar DM, como Hipertensão Arterial, tabagismo, etilismo, ausência de atividade física e sobrepeso e obesidade. Os resultados para fatores de risco dos pacientes diabéticos são apresentados na Figura 2. 
Figura 2 - Fatores de risco em pacientes diabéticos atendidos pelo Ambulatório de Especialidades em Nutrição da URI

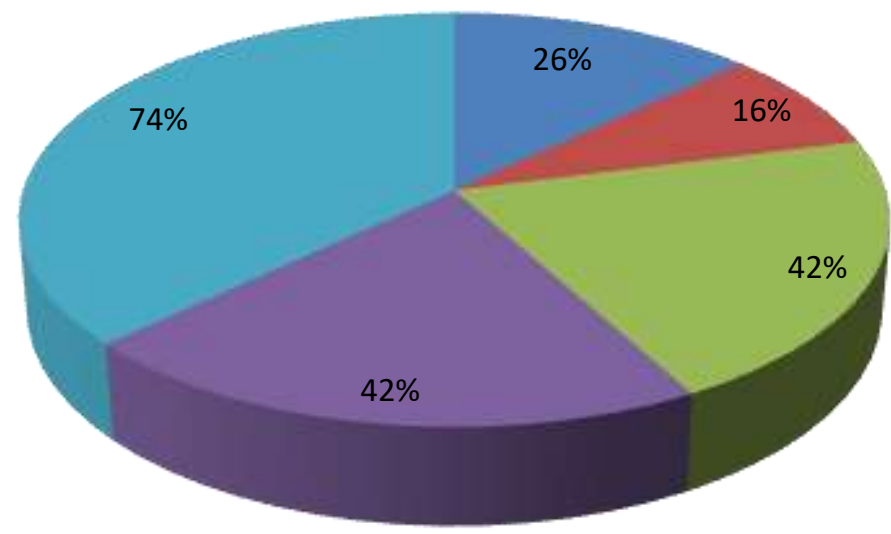

- Hipertensão arterial

- Tabagismo

Etilismo

Atividade Física

Sobrepeso/obesidade

Fonte: MARCHIORO et al, 2017.

Em relação aos fatores de risco, o presente estudo destaca uma elevada frequência de pacientes com sobrepeso e obesidade, afetando em torno de $74 \%$ dos pacientes com DM, o que também pode ser identificado no estudo realizado por Flor et al (2015), onde a prevalência de sobrepeso e obesidade foram atribuídas respectivamente a 49,2\% e 58,3\% da população estudada, sendo a grande parte da carga desta patologia conferida aos fatores de risco modificáveis, enfatizando que, valores elevados de IMC têm causado diversos desfechos desfavoráveis em saúde em diversos países.

Ainda referente aos fatores de risco, este estudo apresenta a falta de atividade física e etilismo com $42 \%$ dos pacientes pesquisados. Em relação a falta de atividade física podemos citar um estudo de Baldo et al (2015), onde os autores afirmam que o tratamento e controle do DM são complexos e envolvem mudanças no estilo de vida do paciente, ainda mais quando os cuidados estão relacionados com a prática regular de atividade física, a automonitoração da glicemia, a administração de medicamentos e a adoção de uma alimentação saudável. Já referente ao etilismo, Lade et al (2016), apontam que o consumo excessivo de álcool por diabéticos aumenta o risco de hipoglicemia tardia, além de prejudicar o controle ponderal e acelerar complicações relacionadas à doença. Outro estudo (Costa; Nascimento, 2014) refere à relevância ao consumo de bebidas alcoólicas como casual em $95,7 \%$ dos pacientes diabéticos pesquisados. Segundo a Sociedade Brasileira de Diabetes (2013), além de o etilismo provocar hipoglicemia e em longo prazo a hipertrigliceridemia, pode resultar em esteatose hepática.

Por fim, na atual pesquisa, porém em menor proporção, os pacientes que apresentam diagnóstico de Hipertensão Arterial como fator de risco representaram $26 \%$ da população pesquisada, e pacientes tabagistas evidenciaram 16\% dos pacientes diabéticos. 
Diferentemente de Lade et al (2016), que aponta o tabagismo com $13 \%$ e o etilismo com $9 \%$ dos pacientes diabéticos em sua pesquisa, demonstrando que existe uma estreita relação entre o tabagismo e doença coronariana, sendo a sua interrupção um fator preponderante na redução do risco de eventos cardiovasculares.

Machado e Campos (2014), descrevem que em pacientes diabéticos, a Hipertensão Arterial é duas vezes mais frequente do que na população em geral. Sendo assim, segundo os autores, o efeito do controle das taxas de glicemia e pressão arterial, provem da adesão adequada do paciente ao tratamento e de práticas de saúde que estimulem ou facilitem a mudança do estilo de vida.

Outro resultado de grande importância para o estudo é a análise do Recordatório Alimentar de 24 horas, onde podemos identificar os hábitos alimentares dos pacientes diabéticos, uma vez que neste questionário é solicitado ao paciente que informe os alimentos que consumiu no dia anterior, bem como, o horário e a quantidade destes alimentos. Com isso é possível analisar o consumo alimentar, a variedade, a frequência de refeições e quantidade dos alimentos consumidos, já que este grupo de pacientes necessita de uma alimentação específica, tendo como preferência o consumo de alimentos integrais e baixo consumo de carboidratos simples, boas fontes de proteínas e lipídeos, além de uma alta ingestão de fibras, entre outros aspectos importantes para manter níveis de glicemia adequados e uma boa resposta ao seu tratamento.

Tabela 6 - Resultados da análise do Recordatório Alimentar 24 horas

\begin{tabular}{l|l|l|l}
\hline Nutrientes & G & $\%$ & Valor de referência \\
\hline Calorias/dia & $1459,785 \pm 584,5669$ & - & - \\
\hline Carboidratos & $214,3932 \pm 95,25808$ & $57,6 \pm 10,26$ & $45-60 \%$ \\
\hline Proteínas & $63,92684 \pm 30,61784$ & $18,68 \pm 9,55$ & $15-20 \%$ \\
\hline Lipídios & $39,21632 \pm 18,97712$ & $23,15 \pm 6,88$ & $30 \%$ \\
\hline
\end{tabular}

Fonte: MARCHIORO et al, 2017.

$\mathrm{Na}$ Tabela 1 foram analisados os nutrientes consumidos por pacientes diabéticos atendidos no Ambulatório de Especialidades em Nutrição da URI - Campus de Frederico Westphalen/RS e constatado que no grupo dos carboidratos a média de consumo é de 57,6 $\pm 10,26 \%$, seguindo com $18,68 \pm 9,55 \%$ de proteínas e $23,15 \pm 6,88 \%$ de lipídeos em suas ingestões diárias.

Segundo o que preconiza as Diretrizes da Sociedade Brasileira de Diabetes (2009), os pacientes diabéticos devem considerar as necessidades individuais e utilizar parâmetros semelhantes à população geral, em todas as faixa etárias, sendo para carboidratos totais de $45-60 \%$, proteína de $15-20 \%$ e lipídeos em torno de $30 \%$.

Como podem ser observados, os resultados da atual pesquisa corroboram com os dados preconizados pela Sociedade Brasileira de Diabetes (2013), onde somente os lipídeos apresentam uma pequena diferença devido à média de consumo recomendado, que é maior do que a apresentado pelos pacientes diabéticos que procuraram o serviço do Ambulatório de Especialidade em Nutrição da URI - Campus de Frederico Westphalen/RS.

Segundo Pontieri, Bachion (2010), as recomendações parecem ser simples e fáceis 
de serem seguidas, porém, dependendo dos hábitos alimentares anteriores destes pacientes, a limitação das quantidades dos nutrientes pode representar uma mudança drástica e originar crenças acerca da terapia nutricional que podem ser barreira à adesão por remeter a práticas restritivas.

\section{CONSIDERAÇÕES FINAIS}

Constatou-se que para obtenção de um bom controle da glicemia e consequentemente uma adequada resposta ao tratamento de DM, os pacientes devem obter uma ingestão de dieta adequada (macro e micronutrientes), e aderir hábitos de vida saudáveis, como por exemplo, evitar bebida alcoólica e fumo e praticar atividade física, além de manter o peso adequado. Estes fatores ajudam a diminuir o risco de complicações da patologia estudada, no caso, o Diabete Mellitus.

Ainda, o portador de DM deve estar sensibilizado sobre a importância de se promover saúde para melhorar sua vida cotidiana. A atuação do profissional nutricionista no cuidado à saúde do paciente diabético é de extrema importância, devido as relevantes atribuições do profissional no acompanhamento destes pacientes, seja com orientações nutricionais específicas, elaboração de plano alimentar, acompanhamento do estado nutricional, entre outras intervenções que o nutricionista pode disponibilizar ao paciente diabético.

\section{REFERÊNCIAS}

BALDO, Cristiano; et al. Diabetes Food Control- Um aplicativo móvel para avaliação do consumo alimentar de pacientes diabéticos. Rev. Eletron. De Comun. Inf. Inov. Saúde, v. 9. n. 3, 2015.

BRASIL. Diretrizes da Sociedade Brasileira de Diabete. Rio de Janeiro, $3^{a}$ edição, p. 400, 2009.

CORTEZ, Daniel Nogueira; et al. Complicações e o tempo de diagnostico do diabetes mellitus na atenção primaria. Acta Paul Enferm. v. 28. n. 3. p. 5-250, 2015.

COSTA, Denise G. S. de P. da; NASCIMENTO, Maria A. B. do. Avaliação do padrão alimentar de indivíduos com diabetes tipo 1 que realizam contagem de carboidratos em uma unidade de saúde pública de Brasília- DF. Com. Ciências Saúde, v. 29. n. 3/4. p. 223-226, 2014.

FLOR, Luísa Sorio; et al. Carga de diabetes no Brasil: fração atribuível ao sobrepeso, obesidade e excesso de peso. Revista Saúde Pública, v. 49 n. 29, 2015.

ISER, Betine Pinto Moehlecke; et al. Prevalência de diabetes autorreferido no Brasil: resultados da Pesquisa Nacional de Saúde 2013. Epidemiol. Serv. Saúde, Brasília, v. 24. n. 2. p. 305-314, 2015. 
INTERNATIONAL DIABETES FEDERATION . IDF Diabetes Atlas. 6th ed. Brussels, Belgium: International Diabetes Federation; 2013.

LADE, Carlos Gabriel de; et al. Análise de Indicadores de Saúde de pacientes com diabetes atendidos pelo Centro Hiperdia de Viçosa. O Mundo da Saúde, São Paulo, v. 40. n. 3. p. 283-292, 2016.

MACHADO, Luis Eduardo; CAMPOS, Renata. O impacto da diabetes melito e da hipertensão arterial para a saúde pública. Saúde Meio Ambient. v. 3. n. 2. p. 53-61, jul./dez. 2014.

MAHAN, L.K; ESCOTT-STUMP, S. RAYMUNDO, J. L. Krause Alimentos, Nutrição e Dietoterapia. In: FRANZ, Marion J.; et al. Terapia Nutricional Clinica para Diabetes Melito e Hipoglicemia de Origem não Diabética. Rio de Janeiro: Elsvier, 13aㅡ ed. cap 31. p. 675-706, 2012.

ORGANIZAÇÃO MUNDIAL DA SAÚDE. Índice de Massa Corporal. 1998. IN: Leão, Leila S. C. de S.; Gomes, Maria do Carmo Rebello. Manual de Nutrição Clínica. Petrópolis, RJ. Vozes. 2012. p. 15.

PONTIERI, Flavia Melo; BACHION, Maria Márcia. Crenças de pacientes diabéticos acerca da terapia nutricional e sua influência na adesão ao tratamento. Ciência \& Saúde Coletiva, v. 1. n. 15. p.151-160, 2010.

SARTORELLI, Daniela Saes; FRANCO, Laercio Joel. Tendências do diabetes mellitus no Brasil: o papel da transição nutricional. Cad. Saúde Pública, Rio de Janeiro, v. 19. n. 1. p. 29-36, 2003.

SOCIEDADE BRASILEIRA DE DIABETES. Diretrizes da Sociedade Brasileira de Diabetes, p. 385, 2013.

TIECHER, Camila Vieira; NASCIMENTO, Maria A. B. do; Controle glicêmico de diabéticos tipo 2 com contagem de carboidratos: uma revisão da literatura. Com. Ciências da Saúde, v. 25. n. 2. p. 149-156, 2014.

TRATAMENTO E ACOMPANHAMENTO DO DIABETES MELLITUS. Diretrizes da Sociedade Brasileira de Diabetes Sociedade Brasileira de Diabetes. Princípios para Orientação Nutricional no Diabetes Mellitus, cap. 5. p. 39, 2009. 